\title{
SHARP MAXIMAL INEQUALITIES FOR CONDITIONALLY SYMMETRIC MARTINGALES AND BROWNIAN MOTION
}

\author{
GANG WANG
}

(Communicated by Lawrence F. Gray)

Abstract. Let $B=\left(B_{t}\right)_{t \geq 0}$ be a standard Brownian motion. For $c>0$, $k>0$, let

$$
\begin{aligned}
T(c, k) & =\inf \left\{t \geq 0: \max _{s \leq t} B_{s}-c B_{t} \geq k\right\}, \\
T^{*}(c, k) & =\inf \left\{t \geq 0: \max _{s \leq t}\left|B_{s}\right|-c\left|B_{t}\right| \geq k\right\} .
\end{aligned}
$$

We show that for $c>0$ and $k>0$, both $T(c, k)$ and $T^{*}(c, k)$ are finite almost everywhere. Moreover, $T(c, k)$ and $T^{*}(c, k) \in L^{p / 2}$ if and only if $c<p /(p-1)$ for $p>1$, and for all $c>0$ when $p \leq 1$. These results have analogues for simple random walks. As a consequence, if $T$ is any stopping time of $B_{t}$ such that $\left(B_{T \wedge t}\right)_{t \geq 0}$ is uniformly integrable, then both of the inequalities

$$
\begin{aligned}
\left\|\sup _{s \leq T} B_{s}\right\|_{p} & \leq \frac{p}{p-1}\left\|B_{T}\right\|_{p}, \\
\left\|\sup _{s \leq T}\left|B_{s}\right|\right\|_{p} & \leq \frac{p}{p-1}\left\|B_{T}\right\|_{p},
\end{aligned}
$$

are sharp. This implies that $q=p /(p-1)$ is not only the best constant for Doob's maximal inequality for general martingales but also for conditionally symmetric martingales (in particular, for dyadic martingales), and for Brownian motion.

\section{INTRODUCTION AND SUMMARY OF RESULTS}

Let $f=\left(f_{0}, f_{1}, \ldots\right)$ be a martingale on a probability space $(\Omega, \mathscr{A}, P)$, and $d=\left(d_{0}, d_{1}, \ldots\right)$ its difference sequence: $d_{0}=f_{0}$ and $d_{n}=f_{n}-f_{n-1}$ for $n \geq 1$. A martingale $f=\left(f_{0}, f_{1}, \ldots\right)$ is conditionally symmetric if $d_{n}$ and $-d_{n}$ have the same distribution given $d_{0}, \ldots, d_{n-1}$ for any $n \geq 1$. Any dyadic martingale is conditionally symmetric. An even more special example of a conditionally symmetric martingale is simple random walk. We say $\left(S_{n}\right)_{n \geq 0}$ is a simple random walk of step $\delta>0$ starting at $a$, if $X_{0}=a$ and $S_{n}=$ $\sum_{i=0}^{n} X_{i}$, where $\left(X_{n}\right)_{n \geq 1}$ is a sequence of independent identically distributed

Received by the editors January 17, 1990.

1980 Mathematics Subject Classification (1985 Revision). Primary 60G42, $60 \mathrm{~J} 65$.

Key words and phrases. Martingale, conditionally symmetric martingale, dyadic martingale, Brownian motion, martingale maximal function. 
random variables with $P\left(X_{1}= \pm \delta\right)=\frac{1}{2}$. For any stopping time $T$ of $\left(S_{n}\right)_{n \geq 0}$, the sequence $f=\left(S_{n \wedge T}\right)_{n \geq 0}$ is a conditionally symmetric martingale, where $a \wedge b=\min (a, b)$. We use $M_{n}(f)$ and $f_{n}^{*}$ to denote $\max _{i \leq n} f_{i}$ and $\max _{i \leq n}\left|f_{i}\right|$ respectively. Note that $\left|M_{n}(f)\right| \leq f_{n}^{*}$. We sometimes use $M_{n}$ to stand for $M_{n}(f)$.

For $p>1$, let $q=p /(p-1)$ be the conjugate of $p$. Recall Doob's inequality [7].

Theorem A. Let $f=\left(f_{0}, f_{1}, \ldots\right)$ be a martingale, then for $p>1, n \geq 1$,

$$
\left\|f_{n}^{*}\right\|_{p} \leq q\left\|f_{n}\right\|_{p} .
$$

It is well known that $q$ is the best constant since it is clearly the best constant in Hardy's inequality, a special case, see for example, Chatterji [4]. Thus, it would be interesting to know whether $q$ is still the best possible constant for conditionally symmetric martingales. The analogue of Theorem A for a continuous time martingale is the following:

Theorem B. Let $f=\left(f_{t}\right)_{t \geq 0}$ be a continuous time martingale with right-continuous paths. If $T$ is any stopping time of $f$ such that $\left(f_{T \wedge t}\right)_{t \geq 0}$ is uniformly integrable, then for $p>1$,

$$
\left\|f_{T}^{*}\right\|_{p} \leq q\left\|f_{T}\right\|_{p} .
$$

It would be interesting to know whether this inequality is sharp for Brownian motion, hence for general continuous time martingales.

We will give positive answers to all of the above questions. In order to state the results of this paper, we introduce some notation.

Let $S=\left(S_{n}\right)_{n \geq 0}$ be a simple random walk of step 1 starting at 0 . We define

$$
\begin{aligned}
N(c, k) & =\inf \left\{n \geq 0: M_{n}(S)-c S_{n} \geq k\right\}, \\
N^{*}(c, k) & =\inf \left\{n \geq 0: S_{n}^{*}-c\left|S_{n}\right| \geq k\right\} .
\end{aligned}
$$

Likewise, for Brownian motion $B=\left(B_{t}\right)_{t \geq 0}$, let

$$
\begin{aligned}
T(c, k) & =\inf \left\{t \geq 0: M_{t}(B)-c B_{t} \geq k\right\}, \\
T^{*}(c, k) & =\inf \left\{t \geq 0: B_{t}^{*}-c\left|B_{t}\right| \geq k\right\} .
\end{aligned}
$$

The main results of this paper are:

Theorem 1. For any $c>0$ and $k>0, N(c, k)<\infty$ and $N^{*}(c, k)<\infty$ a.e. Moreover, $N(c, k) \in L^{p / 2}$ if and only if $c<q$ when $p>1$, and $c<\infty$ when $0<p \leq 1$. Similarly, $N^{*}(c, k) \in L^{p / 2}$ if and only if $c<q$ when $p>1$, and $c<\infty$ when $0<p \leq 1$.

As consequences of Theorem 1, we will show the following:

Theorem 2. For any $c>0$ and $k>0, T(c, k)<\infty$ and $T^{*}(c, k)<\infty$ a.e. Moreover, $T(c, k) \in L^{p / 2}$ if and only if $c<q$ when $p>1$, and $c<\infty$ when 
$0<p \leq 1$. Similarly, $T^{*}(c, k) \in L^{p / 2}$ if and only if $c<q$ when $p>1$, and $c<\infty$ when $0<p \leq 1$.

Combining above results with Theorems $\mathrm{A}$ and $\mathrm{B}$, we get

Theorem 3. Let $f=\left(f_{n}\right)_{n \geq 0}$ be a conditionally symmetric martingale. Then for $p>1, n \geq 1$, both of the inequalities

$$
\left\|f_{n}^{*}\right\|_{p} \leq q\left\|f_{n}\right\|_{p}, \quad\left\|M_{n}\right\|_{p} \leq q\left\|f_{n}\right\|_{p},
$$

are sharp.

Theorem 4. Let $B=\left(B_{t}\right)_{t \geq 0}$ be a standard Brownian motion. If $T$ is a stopping time of $B$ such that $\left(B_{T \wedge t}\right)_{t \geq 0}$ is uniformly integrable, then both of the inequalities

$$
\left\|B_{T}^{*}\right\|_{p} \leq q\left\|B_{T}\right\|_{p}, \quad\left\|M_{T}\right\|_{p} \leq q\left\|B_{T}\right\|_{p},
$$

are sharp.

Thus, the best constant for Doob's general martingale maximal inequality is also the best constant for conditionally symmetric martingales and Brownian motion. This is similar to the behavior of the best constants in the Burkholder inequalities for martingale transforms [3]. In contrast, the constants in Burkholder's square-function inequalities for general martingales [1] are not the same as the best constants for conditionally symmetric martingales or Brownian motion, although for the last cases they are the same. See Davis [6] and Wang [11] for details.

\section{THE PROOF OF THEOREM 1}

It is well known that (see Chung [5], for example), for some integer $c>0$ and all $m$,

$$
P\left(S_{n}=\mathrm{cm} \text { i.o. }\right)=1 \text {. }
$$

In particular, if $k$ is a positive real number, then

$$
P\left(S_{n}=0 \text { i.o. }\right)=1 \quad \text { and } \quad P\left(S_{n} \geq k \text { i.o. }\right)=1 .
$$

Hence $N(c, k)<\infty$ a.e.

Before proving the rest of the theorem, we state a lemma that shows the relationship between $N(c, k)$ and $N^{*}(c, k)$.

Lemma 1. If $k$ is a positive integer, then

$$
N^{*}(c, k)=V_{k}+N(c, k) \circ \theta_{V_{k}} \cdot 1\left(S_{V_{k}}=k\right)+\tilde{N}(c, k) \circ \theta_{V_{k}} \cdot 1\left(S_{V_{k}}=-k\right),
$$

where $V_{k}=\inf \left\{n \geq 0:\left|S_{n}\right|=k\right\}$ and $\tilde{N}(c, k)=\inf \left\{n \geq 0: c S_{n}-\inf _{i \leq n} S_{i} \geq k\right\}$. The function $\theta$ is the shift transformation: $\Omega \rightarrow \Omega$ obtained by setting $\theta_{n}(\omega)(m)$ $=\omega(m+n)$ for $m, n \geq 0$.

Note that $N(c, k)$ and $\tilde{N}(c, k)$ have the same distribution. 
Proof. Since $V_{k}<\infty$ a.e., either $S_{V_{k}}=k$ or $S_{V_{k}}=-k$.

Because $k>0$, we have $V_{k} \geq 1$. Let $m \geq 1$. On $\left(S_{V_{k}}=k, V_{k}=m\right)$, when $i<m, S_{i}^{*}-c\left|S_{i}\right| \leq S_{i}^{*}<k$. So $N^{*}(c, k) \geq m$. Taking $m \leq i \leq N^{*}(c, k)$, we claim $S_{i} \geq 0$. Otherwise, there exists an $m \leq i_{0}<N^{*}(c, k)$ such that $S_{i_{0}}=0$ since $S_{m}=k>0$. Then, $S_{i_{0}}^{*}-c\left|S_{i_{0}}\right| \geq S_{m}^{*}=k$, contrary to $i_{0}<N^{*}(c, k)$. Hence, $S_{i} \geq 0$ for $m \leq i \leq N^{*}(c, k)$. Consequently, by $S_{m}^{*}=M_{m}=S_{m}=k$,

$$
\begin{aligned}
N^{*}(c, k) & =\inf \left\{n \geq m: M_{n}(S)-c S_{n} \geq k\right\} \\
& =m+\inf \left\{n \geq 0: M_{n}(S) \circ \theta_{m}-c S_{n} \circ \theta_{m} \geq k\right\} \\
& =m+N(c, k) \circ \theta_{m} .
\end{aligned}
$$

Thus $N^{*}(c, k)=V_{k}+N(c, k) \circ \theta_{V_{k}}$ on $\left(S_{V_{k}}=k\right)$. Similarly, $N^{*}(c, k)=$ $V_{k}+\tilde{N}(c, k) \circ \theta_{V_{k}}$ on $\left(S_{V_{k}}=-k\right)$. This completes the proof.

We shall show that, if $c>0$ and $k>0$, then $N(c, k) \in L^{p / 2}$ if and only if $c<q$ when $p>1$ and $c<\infty$ when $0<p \leq 1$. Then we apply Lemma 1 to get similar results for $N^{*}(c, k)$.

We start with another lemma.

Lemma 2. Let $x$ be any positive integer, then

$$
N(c, k)=R_{x}+N(c, k) \circ \theta_{R_{x}} \text { on } R_{x} \leq N(c, k),
$$

where $R_{x}=\inf \left\{n \geq 0: S_{n}=x\right\}$.

Proof. On $R_{x} \leq N(c, k)$, since $M_{R_{x}}(S)=S_{R_{x}}=x$,

$$
\begin{aligned}
N(c, k) & =\inf \left\{n \geq R_{x}: M_{n}(S)-c S_{n} \geq k\right\} \\
& =R_{x}+\inf \left\{n \geq 0, M_{n}(S) \circ \theta_{R_{x}}-c S_{n} \circ \theta_{R_{x}} \geq k\right\} \\
& =R_{x}+N(c, k) \circ \theta_{R_{x}} \cdot \square
\end{aligned}
$$

Now we state the main result. Here $\lceil x\rceil=\inf \{k: k$ is integer, $k \geq x\}$.

Corollary 2.1. Let $c \geq 1$. If $x$ be any positive integer, then

$$
P\left(M_{N} \geq x\right)=f(c, x) \cdot P\left(M_{N} \geq x-1\right),
$$

where $N=N(c, k)$, and

$$
f(c, x)=\frac{\lceil(k+(c-1)(x-1)) / c\rceil}{\lceil(k+(c-1)(x-1)) / c\rceil+1} .
$$

Proof. Since $R_{x}=R_{x-1}+R_{x} \circ \theta_{R_{x-1}}$, we have, by Lemma 2,

$$
\begin{aligned}
P\left(M_{N} \geq x\right) & =P\left(R_{x} \leq N\right) \\
& =P\left(R_{x-1} \leq N, R_{x} \leq N\right) \\
& =P\left(R_{x-1} \leq N, R_{x-1}+R_{x} \circ \theta_{R_{x-1}} \leq N\right) \\
& =P\left(R_{x-1} \leq N, R_{x-1}+R_{x} \circ \theta_{R_{x-1}} \leq R_{x-1}+N \circ \theta_{R_{x-1}}\right) \\
& =P\left(R_{x-1} \leq N, R_{x} \circ \theta_{R_{x-1}} \leq N \circ \theta_{R_{x-1}}\right) \\
& =P\left(R_{x-1} \leq N\right) P\left(R_{x}^{\prime} \leq N_{x}\right) \\
& =P\left(M_{N} \geq x-1\right) P\left(M_{N_{x}}\left(S^{\prime}\right) \geq x\right),
\end{aligned}
$$


where $N_{x}=N_{x}(c, k)=\inf \left\{n \geq 0: M_{n}\left(S^{\prime}\right)-c S_{n}^{\prime} \geq k\right\}$ and where $R_{x}^{\prime}=$ $\inf \left\{n \geq 0: S_{n}^{\prime}=x\right\}$. The sequence $S^{\prime}=\left(S_{n}^{\prime}\right)_{n \geq 0}$ with $S_{n}^{\prime}=x-1+S_{n}$ is a simple random walk of step 1 starting at $x-1$. The next to last equality is from the strong Markovian property of $S$. We now show $f(c, x)=P\left(M_{N_{x}}\left(S^{\prime}\right) \geq\right.$ $x)=\lceil(k+(c-1)(x-1)) / c\rceil /(\lceil(k+(c-1)(x-1)) / c\rceil+1)$.

First we show that $N_{x}(c, k)$ and $N(c, k+(c-1)(x-1))$ have the same distribution.

By $S_{n}^{\prime}=x-1+S_{n}$, we see, for any natural number $m$,

$$
\begin{aligned}
P\left(N_{x}(c, k)=m\right)= & P\left(\left(M_{m}(S)+x-1\right)-c\left(S_{m}+x-1\right) \geq k,\right. \\
& \left.\left(M_{i}(S)+x-1\right)-c\left(S_{i}+x-1\right)<k, \quad i<m\right) \\
= & P\left(M_{m}(S)-c S_{m} \geq k+(c-1)(x-1),\right. \\
& \left.M_{i}(S)-c S_{i}<k+(c-1)(x-1), \quad i<m\right) \\
= & P(N(c, k+(c-1)(x-1))=m) .
\end{aligned}
$$

So they have the same distribution. Thus $N_{x}<\infty$ a.e. Hence,

$$
\begin{aligned}
P\left(M_{N_{x}}\left(S^{\prime}\right) \geq x\right) & =1-P\left(M_{N_{x}}\left(S^{\prime}\right) \leq x-1\right) \\
& =1-\sum_{m=0}^{\infty} P\left(M_{m}\left(S^{\prime}\right) \leq x-1, N_{x}=m\right) .
\end{aligned}
$$

Using $S_{m}^{\prime}=x-1+S_{m}$ and $M_{m}(S) \geq 0$, we have, for any $m \geq 0$,

$$
\begin{aligned}
& P\left(M_{m}\left(S^{\prime}\right) \leq x-1, N_{x}=m\right) \\
& \quad=P\left(M_{m}(S) \leq 0, M_{m}(S)-c S_{m} \geq k+(c-1)(x-1),\right. \\
& \left.\quad M_{i}(S)-c S_{i}<k+(c-1)(x-1), i<m\right) \\
& \quad P\left(M_{m}(S)=0, S_{m} \leq-\frac{k+(c-1)(x-1)}{c},\right. \\
& \left.\quad S_{i}>-\frac{k+(c-1)(x-1)}{c}, i<m\right) \\
& =P\left(R_{k^{0}}^{1}=m, S_{R^{1}}=k^{0}\right),
\end{aligned}
$$

where $R_{b}^{a}=\inf \left\{n \geq 0: S_{n} \geq a\right.$ or $\left.S_{n} \leq b\right\}$ and $k^{0}=-\lceil(k+(c-1)(x-1)) / c\rceil$. Thus, by (1),

$$
P\left(M_{N_{x}}\left(S^{\prime}\right) \geq x\right)=1-P\left(S_{R_{k^{0}}^{1}}=k^{0}\right)=\frac{\lceil(k+(c-1)(x-1)) / c\rceil}{\lceil(k+(c-1)(x-1)) / c\rceil+1} .
$$

This finishes the proof.

Remark. When $c=1, f(c, x)=k /(k+1)$. Thus $M_{N}$ is geometrically distributed. Dubins and Schwarz [9] showed a similar result; namely, that $M_{T}=$ $\sup _{t \leq T} B_{t}$ is exponentially distributed, where $T=T(1, k)$. When $c<1$, 
all of the above arguments are still valid up to $P\left(M_{N} \geq x\right)=$ $P\left(M_{N} \geq x-1\right) P\left(M_{N_{x}}\left(S^{\prime}\right) \geq x\right)$. Let $x$ be large enough so that $(1-c)(x-1) \geq$ $k$, then $N_{x}=0$. Consequently, $M_{N_{x}}\left(S^{\prime}\right)=M_{0}\left(S^{\prime}\right)=x-1<x$. So $P\left(M_{N} \geq x\right)=0$. This means $M_{N}$ is bounded. Hence, when $c \leq 1, S_{N}^{*} \in L^{p}$ for all $p>0: S_{N}^{*} \leq M_{N}+(k+1) / c$ where $S_{N \wedge n} \geq-(k+1) / c$ for all positive integers $n$. Note that $N^{1 / 2}=N^{1 / 2}(c, k)$ is the square-function of the martingale $\left(S_{N \wedge n}\right)_{n \geq 0}$ and $S_{N}^{*}$ is its maximal function. Thus, by the square-function inequality for martingales (Burkholder [1]), we have $N(c, k) \in L^{p}$ for all $p>0$. We therefore have shown the following:

Corollary 2.2. Let $c \leq 1$ and $k>0$, then $N(c, k) \in L^{p}$ for $p>0$.

Corollary 2.1 implies that, for any positive integer $n, P\left(M_{N} \geq n\right)=$ $\prod_{i=1}^{n} f(c, i)$.

Lemma 3. $\ln \left(P\left(M_{N} \geq n\right)\right)=-\frac{c}{c-1} \ln (n)+O(1)$, for $c>1$ and positive integer $n$.

Proof. By calculus,

$$
\begin{aligned}
\ln \left(P\left(M_{N} \geq n\right)\right) & =\sum_{i=1}^{n} \ln (f(c, i))=\sum_{i=1}^{n} \ln \left(\frac{\lceil(k+(c-1)(i-1)) / c\rceil}{\lceil(k+(c-1)(i-1)) / c\rceil+1}\right) \\
& =\sum_{i=0}^{n-1} \ln \left(1-\frac{\frac{c}{c-1}}{i+\frac{k+c}{c-1}}\right)+O(1)=-\frac{c}{c-1} \sum_{i=0}^{n-1} \frac{1}{i+\frac{k+c}{c-1}}+O(1) \\
& =-\frac{c}{c-1} \sum_{i=1}^{n-1} \frac{1}{i}+O(1)=-\frac{c}{c-1} \ln (n)+O(1) . \quad \square
\end{aligned}
$$

As a consequence, we get

Corollary 3.1. Let $c>1$ and $k>0$, then $N(c, k) \in L^{p / 2}$ if and only if $c<q$ for $p>1$ or $c<\infty$ when $p \leq 1$.

Proof. Since $N(c, k)<\infty$ a.e., $M_{N} \in L^{p}$ iff $\sum_{n=1}^{\infty} n^{p-1} P\left(M_{N} \geq n\right)<\infty$. By Lemma 3, this is equivalent to $\sum_{n=1}^{\infty} n^{c_{p}}<\infty$, where $c_{p}=p-1-c /(c-1)$. Thus $p-1-c /(c-1)<-1$, that is, $c<q$ when $p>1$, or $c<\infty$ when $p \leq 1$. But $M_{N}-c S_{N}=\lceil k\rceil$ and $M_{N} \leq S_{N}^{*} \leq M_{N}+(k+1) / c$, we have $S_{N} \in L^{p}$ and $S_{N}^{*} \in L^{p}$ if and only if $c<q$ when $p>1$, or $c<\infty$ when $p \leq 1$. Now by square-function inequality for martingales, we have $N(c, k) \in L^{p / 2}$ if and only if $c<q$ when $p>1$, or $c<\infty$ when $p \leq 1$. This proves the corollary.

Combining Corollaries 2.2 and 3.1, we show the half part of Theorem 1 about $N(c, k)$. For another half, by the strong Markovian property and Lemma 1, we have for $c>0$ and $k>0, N^{*}(c, k)<\infty$ a.e. Meanwhile,

$$
E(N(c, c k))^{p / 2} \leq E\left(N^{*}(c, k)\right)^{p / 2} \leq 2^{p / 2}\left(E(N(c, c k))^{p / 2}+E\left(V_{k}\right)^{p / 2}\right) .
$$

Therefore, $N^{*}(c, k) \in L^{p / 2}$ if and only if $N(c, c k) \in L^{p / 2}$. This completes the proof. 


\section{THE PROOF OF THEOREM 2}

The following is analogous to Lemma 1.

Lemma 4. If $k$ is a natural number, then

$$
T^{*}(c, k)=\nu_{k}+T(c, k) \circ \theta_{\nu_{k}} \cdot 1_{\left(B_{\nu_{k}}=k\right)}+\tilde{T}(c, k) \circ \theta_{\nu_{k}} \cdot 1_{\left(B_{\nu_{k}}=-k\right)},
$$

where $\nu_{k}=\inf \left\{t \geq 0:\left|B_{t}\right|=k\right\}$ and $\widetilde{T}(c, k)=\inf \left\{t \geq 0: c B_{t}-\inf _{s \leq t} B_{s} \geq k\right\}$.

We omit the proof since it is similar to Lemma 1. The reader should notice that $T(c, k)$ and $\widetilde{T}(c, k)$ have the same distribution.

Because Brownian motion can be approximated by simple random walks of small steps starting at 0 , it is natural that the above results should be able to extend to $T(c, k)$ and $T^{*}(c, k)$. First note that Theorem 1 still holds for simple random walks of step size $\delta$ starting at 0 . For a positive integer $j$, we shall follow a well-known procedure to approximate Brownian motion by a simple random walk of step size $1 / j$ starting at 0 . Let $\tau(0, j)=0$. Define $\tau(1, j)=$ $\inf \left\{t \geq 0:\left|B_{t}\right|=1 / j\right\}$ and $\tau(m, j)=\tau(m-1, j)+\tau(1, j) \circ \theta_{\tau(m-1, j)}$ for $m \geq 1$. Then $S^{j}=\left(S_{n}^{j}\right)_{n \geq 0}=\left(B_{\tau(n, j)}\right)_{n \geq 0}$ is a simple random walk of step $1 / j$ starting at 0 . If $N_{j}(c, k)=\inf \left\{n \geq 0: M_{n}\left(S^{j}\right)-c S_{n}^{j} \geq k\right\}$ and $N_{j}^{*}(c, k)=$ $\inf \left\{n \geq 0: S_{n}^{j^{*}}-c\left|S_{n}^{j}\right| \geq k\right\}$, then $Q(c, k, j)=\tau\left(N_{j}(c, k), j\right)$ and $Q^{*}(c, k, j)$ $=\tau\left(N_{j}^{*}(c, k), j\right)$ are stopping times of Brownian motion. By the definition of $\tau(n, j)$, we see, for $n \geq 1$,

$$
\left|B_{t}-S_{n}^{j}\right| \leq 1 / j \quad \text { for } \tau(n-1, j)<t \leq \tau(n, j) .
$$

Moreover, $B_{Q(c, k, j)}=S_{N_{j}(c, k)}^{j}$ and $B_{Q^{*}(c, k, j)}=S_{N_{j}^{*}(c, k)}^{j}$. Consequently,

$$
\begin{gathered}
M_{N_{j}(c, k)}\left(S^{j}\right)-1 / j \leq M_{Q(c, k, j)}(B) \leq M_{N_{j}(c, k)}\left(S^{j}\right)+1 / j, \\
S_{N_{j}^{*}(c, k)}^{j^{*}}-1 / j \leq B_{Q^{*}(c, k, j)}^{*} \leq S_{N_{j}^{*}(c, k)}^{j^{*}}+1 / j .
\end{gathered}
$$

By Theorem 1, these show

$$
\begin{gathered}
T(c, k-1 / j) \leq Q(c, k, j)<\infty \\
T^{*}(c, k-1 / j) \leq Q^{*}(c, k, j)<\infty \text { a.e. }
\end{gathered}
$$

Since $k$ and $j$ are arbitrary, we have shown that $T(c, k)<\infty$ and $T^{*}(c, k)<$ $\infty$ a.e.

Next, by the Burkholder inequality for Brownian motion ([1,2] or Millar [10]), the facts that $S_{N_{j}(c, k)}^{*^{*}} \leq M_{N_{j}(c, k)}\left(S^{j}\right)+(k+1) / c$ and that $B_{Q(c, k, j)}^{*} \leq$ $M_{Q(c, k, j)}(B)+(k+2) / c$ from (2), (3), and (4), we see that when $p>1$, $Q(c, k, j)$ and $Q^{*}(c, k, j) \in L^{p / 2}$ if and only if $N_{j}(c, k)$ and $N_{j}^{*}(c, k) \in$ $L^{p / 2}$ respectively. Thus, using Theorem 1 and (5), we have $T(c, k) \in L^{p / 2}$ and $T^{*}(c, k) \in L^{p / 2}$ if $c<q$ when $p>1$. This implies that $T(c, k) \in L^{p / 2}$ and $T^{*}(c, k) \in L^{p / 2}$ if $c<q$ when $p>1$ and $c<\infty$ when $p \leq 1$. To see that $T(c, k) \notin L^{p / 2}$ and $T^{*}(c, k) \notin L^{p / 2}$ when $c \geq q$, note that by Lemma 4 and the strong Markovian property of Brownian motion, we have $T^{*}(c, k) \in L^{p / 2}$ 
if and only if $T(c, c k) \in L^{p / 2}$. Thus we may assume $T^{*}(c, k) \in L^{p / 2}$. Then, by Burkholder inequality,

$$
\left\|B_{T^{*}(c, k)}^{*}\right\|_{p}=\left\|c\left|B_{T^{*}(c, k)}\right|+k\right\|_{p}>q\left\|B_{T^{*}(c, k)}\right\|_{p}
$$

which is contrary to Theorem $\mathrm{B}$.

This completes the proof of Theorem 2 .

\section{THE PROOFS OF THEOREMS 3 AND 4}

We need to show only that for any $1<c<q$,

$$
\begin{gathered}
\left\|S_{N^{*}(c, k)}^{*}\right\|_{p} \geq c\left\|S_{N^{*}(c, k)}\right\|_{p}, \\
\left\|M_{N(c, k)}(S)\right\|_{p} \geq c\left\|S_{N(c, k)}\right\|_{p} .
\end{gathered}
$$

These imply

$$
\begin{gathered}
\left\|B_{Q^{*}(c, k, 1)}^{*}\right\|_{p} \geq\left\|S_{N^{*}(c, k)}^{*}\right\|_{p} \geq c\left\|B_{Q^{*}(c, k, 1)}\right\|_{p}, \\
\left\|M_{Q(c, k, 1)}(B)\right\|_{p} \geq\left\|M_{N(c, k)}(S)\right\|_{p} \geq c\left\|B_{Q(c, k, 1)}\right\|_{p} .
\end{gathered}
$$

From the definition of $N^{*}(c, k),(6)$ clearly follows. To show (7), note that when $k \leq 1$ and $1<c<q, S_{N(c, k)}=\left(M_{N(c, k)}-1\right) / c \geq-1 / c>-1$. Hence, $S_{N(c, k)} \geq 0$. Thus, from the definition of $N(c, k)$, (7) follows. This completes the proofs.

\section{ACKNOWLEDGMENT}

I wish to thank Professor Burkholder for suggesting this problem to me and providing continued guidance. I also wish to express my gratitude to Professor Davis and Professor Bañuelos for invaluable discussions.

\section{REFERENCES}

1. D. L. Burkholder, Martingale transforms, Ann. Math. Stat. 37 (1966), 1494-1505.

2. __ Distribution function inequalities for martingales, Ann. Probab. 1 (1973), 19-42.

3. _ Boundary value problems and sharp inequalities for martingale transforms, Ann. Probab. 12 (1984), 647-702.

4. S. D. Chatterji, Les martingales et leurs applications analytiques, Lecture Notes in Math., vol. 307, Springer-Verlag, Berlin, 1973.

5. K. L. Chung, $A$ course in probability theory, Academic Press, New York, 1974.

6. B. Davis, On the $L^{p}$ norm of stochastic integrals and other martingales, Duke Math. J. 43 (1976), 697-704.

7. J. L. Doob, Stochastic processes, Wiley, New York, 1951.

8. L. E. Dubins and D. Gilat, On the distribution of maxima of martingales, Proc. Amer. Math. Soc. 68 (1978), 337-338.

9. L. E. Dubins and G. Schwarz, A sharp inequality for sub-martingales and stopping-times, Colloque Paul Lévy, Astérisque 157-158 (1988), 129-145.

10. P. W. Millar, Martingale integrals, Amer. Math. Soc. Transl. Ser. 2133 (1968), 145-166.

11. G. Wang, Some sharp inequalities for conditionally symmetric martingales, Ph.D. thesis, University of Illinois at Urbana-Champaign, 1989.

Department of Mathematics, Purdue University, West Lafayette, Indiana 47907 